\title{
Evaluation of High-sensitivity C-reactive Protein and Lipid Profile in Nondiabetic Siblings and Offspring of Type 2 Diabetes Mellitus Patients
}

\author{
Kamalakar B Mane ${ }^{1}$, Shilpa Asegaonkar²
}

\begin{abstract}
Introduction: Onset of type 2 diabetes mellitus (T2DM) at early age heralds many years of disease and an increased risk that the full range of both microvascular and macrovascular complications will occur when affected individuals are still relatively young. Thus, further generations may be burdened with morbidity and mortality at the height of their productivity, potentially affecting workface, and healthcare systems of countries across the world.

Materials and methods: The present study carried out at Department of Biochemistry at tertiary care hospital; total 100 nondiabetic siblings and offspring of T2DM patients between the age group 20 and 50 years selected on outpatient department (OPD) basis and compared with 100 age- and sex-matched healthy controls. Serum tested for plasma glucose level, serum high-sensitivity C-reactive protein (hs-CRP), serum cholesterol, serum triglycerides (TGs), and high-density lipoprotein (HDL) levels.

Results: The mean value of blood sugar level did not show significant difference between the cases and controls $(92.02 \pm 9.23$ vs $91.77 \pm 7.99$, $p \geq 0.05)$. The mean values of hs-CRP $(2.4 \pm 1.98$ vs $1.0 \pm 0.38)$, TG $(167.35 \pm 17.35$ vs $124.63 \pm 13.55)$, total cholesterol (TC) (176.99 \pm 12.45 vs $147.59 \pm 9.72)$, low-density lipoprotein (LDL) (106.41 \pm 12.99 vs $71.65 \pm 11.24)$, and very high-density lipoprotein (VHDL) (33.47 \pm 3.47 vs $24.93 \pm 2.71)$ (all $p<0.001)$ were increased, however mean value of HDL $(37.11 \pm 3.99$ vs $51.01 \pm 3.93)$ was decreased in the cases as compared to controls. High-sensitivity C-reactive protein shows positive correlation with TG, TC, LDL, and very low-density lipoprotein and has negative correlation with HDL.

Conclusion: Timely screening and early detection of the increased hs-CRP in the first-degree relatives of T2DM subjects may help clinicians enable to intervene early in the course of disease and to prevent further complications and outcomes. Therefore, primary prevention by target screening among high-risk individuals to prevent transition to overt T2DM by therapeutic lifestyle changes is a feasible and attractive alternative to reduce diabetes-related morbidity and mortality.
\end{abstract}

Keywords: High-sensitivity C-reactive protein, Lipid profile, Siblings and offspring, Type 2 diabetes mellitus.

Indian Journal of Medical Biochemistry (2020): 10.5005/jp-journals-10054-0135

\section{INTRODUCTION}

Diabetes mellitus (DM) with its complication has become the most important and challenging contemporary health problem. Globally, the estimated number of adults with diabetes in 2007 was 246 million and 380 million adults worldwide will have diabetes by 2025. India has 41 million diabetics and this number is expected to increase to 70 million by $2025 .{ }^{1}$ Over the past 30 years, the status of diabetes has changed from being considered as a mild disorder of the elderly to one of the major causes of morbidity and mortality affecting the youth and middle-aged people. ${ }^{2}$ Onset of type 2 diabetes mellitus (T2DM) at early age heralds many years of disease and an increased risk that the full range of both microvascular and macrovascular complications will occur when affected individuals are still relatively young. Thus, further generations may be burdened with morbidity and mortality at the height of their productivity, potentially affecting workface, and healthcare systems of countries across the world. ${ }^{3}$

In India, nearly 75\% of T2DM patients have the first-degree family history. The lifetime risk of developing the disease is about $40 \%$ in offspring of one parent with T2DM and the risk approaches to $70 \%$ if both parents have diabetes. In terms of sibling relative risk, a first-degree relative (FDR) of a patient with T2DM has a threefold increased risk of developing the disease. ${ }^{5}$ It is found that Indians have $45 \%$ positive family history of diabetes as compared to $38 \%$
1Department of Biochemistry, Dr VM Government Medical College, Solapur, Maharashtra, India

${ }^{2}$ Department of Biochemistry, Government Medical College, Aurangabad, Maharashtra, India

Corresponding Author: Kamalakar B Mane, Department of Biochemistry, Dr VM Government Medical College, Solapur, Maharashtra, India, Phone:+91 7972584798,e-mail:drkamlakarmane@ gmail.com

How to cite this article: Mane KB, Asegaonkar S. Evaluation of Highsensitivity C-reactive Protein and Lipid Profile in Nondiabetic Siblings and Offspring of Type 2 Diabetes Mellitus Patients. Indian J Med Biochem 2020;24(1):32-36.

Source of support: Nil

Conflict of interest: None

of the Europeans. ${ }^{6}$ Lipid metabolism in T2DM is modulated by a series of factors among which, the degree of glycemic control and the presence of insulin resistance (IR) are the two most important factors. Diabetic dyslipidemia is a complex cluster of potentially atherogenic lipid and lipoprotein changes. Increased plasma triglycerides (TGs), especially very high-density lipoprotein (VLDL), TG, and low concentration of high-density lipoprotein cholesterol (HDL-C), preponderance of small, dense low-density lipoprotein 
(LDL) and excessive postprandial lipemia are the main components of diabetic dyslipidemia. ${ }^{7}$

Diabetic dyslipidemia is often found in prediabetes, i.e., patients with IR with normal indices of plasma glucose. ${ }^{8}$ There are ample evidences supporting the role of chronic, low-grade inflammation in the pathogenesis of IR. Proinflammatory cytokines and acute phase reactants are correlated with clinical features of IR and plasma insulin concentrations. According to American Heart Association (AHA) guidelines 2002, C-reactive protein (CRP) \{as measured by high-sensitivity assay [high-sensitivity C-reactive protein (hs-CRP)]\} is the inflammatory marker of choice, as it has most stability, assay precision, accuracy, and availability. ${ }^{9}$

\section{Materials and Methods}

The present case-control study was carried out at Department of Biochemistry at tertiary care hospital. After approval from institutional ethical committee written and informed consent, total 100 nondiabetic siblings and offspring of T2DM patients between the age group 20 and 50 years were selected on outpatient department (OPD) basis and compared with 100 age- and sexmatched healthy controls. Individuals not having any family history of T2DM among FDRs, T2DM patients, patients suffering from any acute or chronic cardiovascular diseases and any other major illness, and subjects taking oral contraceptives pills were excluded from study. Participants were selected on the basis of detailed history, clinical examination, and laboratory investigations. Detailed history of participants including age, sex, and marital status, history of any medications, addictions, dietary habits, and lifestyle was recorded. Anthropometric measurements, such as body weight (kg), height $(\mathrm{m})$, waist circumference $(\mathrm{cm})$, and hip circumference $(\mathrm{cm})$, were recorded. Body mass index and waist:hip ratios were calculated. After written informed consent, 12 hour fasting venous blood samples were collected from all participants in fluoride and plain bulbs. Serum was separated after 1 hour by centrifugation at 3,000 rpm for 10 minutes and was tested for plasma glucose level (GODPOD method), serum hs-CRP [chemiluminescence immunoassay (CLIA) using Acculite CLIA microwells. Assay kits from Monobind Inc., Lake Forest, CA 92630, USA], serum cholesterol (cholesterol oxidaseperoxidase), serum TGs, and high-density lipoprotein (HDL) levels.

\section{Statistical Analysis}

The results were analyzed by SPSS software, version 10. The results were interpreted as mean + standard deviation (SD). Unpaired t test was applied for comparing between two groups and correlation coefficients were calculated ( $r$ value). $p$ value was obtained from unpaired $t$ test and $<0.05$ was considered statistically significant. Positive and negative $r$ values were estimated to find out the strength of correlation.

\section{Results and Observations}

A total of 200 participants were enrolled in the study, 100 as cases with family history of T2DM and 100 as controls without family history of T2DM.

Demographic and biochemical data of all the participants were analyzed as mean + SD. Correlation coefficients $(r)$ were calculated among various parameters in the cases and controls.

Table 1 represents demographic data of the cases and controls. Mean age $(35.44 \pm 6.18$ vs $36.31 \pm 5.34, p \geq 0.05)$, weight $(60.91 \pm$ 7.28 vs $59.93 \pm 6.96, p \geq 0.05)$, and height ( $1.63 \pm 0.08$ vs $1.63 \pm 0.07$,
Table 1: Comparison of demographic parameters

\begin{tabular}{lccl}
\hline & \multicolumn{3}{c}{ Mean \pm SD and p value } \\
\cline { 2 - 4 } Parameter & Cases $(n=100)$ & Control $(n=100)$ & $p$ value \\
\hline Age (years) & $35.44 \pm 6.18$ & $36.31 \pm 5.34$ & $>0.05$ \\
Sex $(\mathrm{M} / \mathrm{F})$ & $58 / 42$ & $53 / 47$ & - \\
Weight $(\mathrm{kg})$ & $60.91 \pm 7.28$ & $59.93 \pm 6.96$ & $>0.05$ \\
Height $(\mathrm{m})$ & $1.63 \pm 0.08$ & $1.63 \pm 0.07$ & $>0.05$ \\
BMI $\left(\mathrm{kg} / \mathrm{m}^{2}\right)$ & $22.81 \pm 1.27$ & $22.43 \pm 1.02$ & $<0.05$ \\
WC $(\mathrm{cm})$ & $78.98 \pm 5.43$ & $73.97 \pm 4.88$ & $<0.001$ \\
HC $(\mathrm{cm})$ & $98.24 \pm 6.90$ & $96.08 \pm 5.31$ & $<0.05$ \\
W/H ratio & $0.81 \pm 0.07$ & $0.77 \pm 0.04$ & $<0.001$ \\
\hline
\end{tabular}

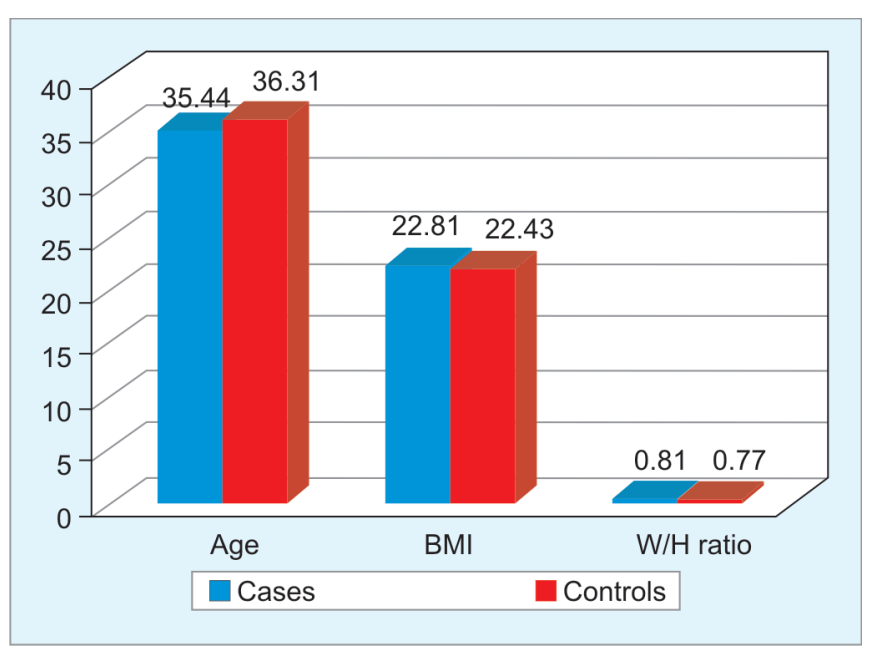

Fig. 1: Graphical comparison of anthropometric parameters

$p>0.05$ ) were not significantly different between the cases and controls. The data showed increase in mean value of body mass index (BMI) $(22.81 \pm 1.27$ vs $22.43 \pm 1.02, p<0.05)$ and hip circumference $(98.24 \pm 6.90$ vs $96.08 \pm 5.31, p<0.05)$ in the cases as compared to controls. The data also showed highly significant difference in mean values of waist circumference ( $78.98 \pm 5.43$ vs $73.97 \pm 4.88, p<0.001)$ and waist/hip ratio $(0.81 \pm 0.07$ vs $0.77 \pm 0.04, p<0.001)$ (Fig. 1).

Table 2 shows comparison of biochemical parameters between the cases and controls. The mean value of blood sugar level did not show significant difference between the cases and controls (92.02 \pm 9.23 vs $91.77 \pm 7.99, p \geq 0.05)$. The mean values of hs-CRP $(2.4 \pm 1.98$ vs $1.0 \pm 0.38)$, TG $(167.35 \pm 17.35$ vs $124.63 \pm 13.55)$, total cholesterol (TC) $(176.99 \pm 12.45$ vs $147.59 \pm 9.72), \mathrm{LDL}(106.41 \pm 12.99$ vs 71.65 $\pm 11.24)$, and very high-density lipoprotein (VHDL) (33.47 \pm 3.47 vs $24.93 \pm 2.71$ ) (all $p<0.001$ ) were increased, however mean value of HDL ( $37.11 \pm 3.99$ vs $51.01 \pm 3.93)$ was decreased in the cases as compared to controls (Fig. 2).

In Table 3, hs-CRP shows positive correlation with TG, TC, LDL, and VLDL and has negative correlation with HDL.

\section{Discussion}

There is growing evidence supporting the concept that chronic, low-grade, inflammatory states may have a pathogenic role in IR. Several studies have shown that proinflammatory cytokines and acute-phase reactants are correlated with measures of IR, BMI, waist circumference, circulating TG, and HDL cholesterol concentration. Inflammatory cytokines, such as tumor necrosis factor-a (TNF-a) 
Table 2: Comparison of biochemical parameters

\begin{tabular}{lccl}
\hline & \multicolumn{3}{c}{ Mean \pm SD and $p$ value } \\
\cline { 2 - 4 } Parameter & Cases $(n=100)$ & Controls $(n=100)$ & $p$ value \\
\hline BSL 70-110 mg/dL & $92.02 \pm 9.23$ & $91.77 \pm 7.99$ & $>0.05$ \\
hs-CRP up to $1 \mathrm{mg} / \mathrm{L}$ & $2.4 \pm 1.98$ & $1.0 \pm 0.38$ & $<0.001$ \\
TG up to $150 \mathrm{mg} \%$ & $167.35 \pm 17.35$ & $124.63 \pm 13.55$ & $<0.001$ \\
TC up to $200 \mathrm{mg} \%$ & $176.99 \pm 12.45$ & $147.59 \pm 9.72$ & $<0.001$ \\
HDL 40-60 mg\% & $37.11 \pm 3.99$ & $51.01 \pm 3.93$ & $<0.001$ \\
LDL up to $100 \mathrm{mg} \%$ & $106.41 \pm 12.99$ & $71.65 \pm 11.24$ & $<0.001$ \\
VLDL & $33.47 \pm 3.47$ & $24.93 \pm 2.71$ & $<0.001$ \\
\hline
\end{tabular}

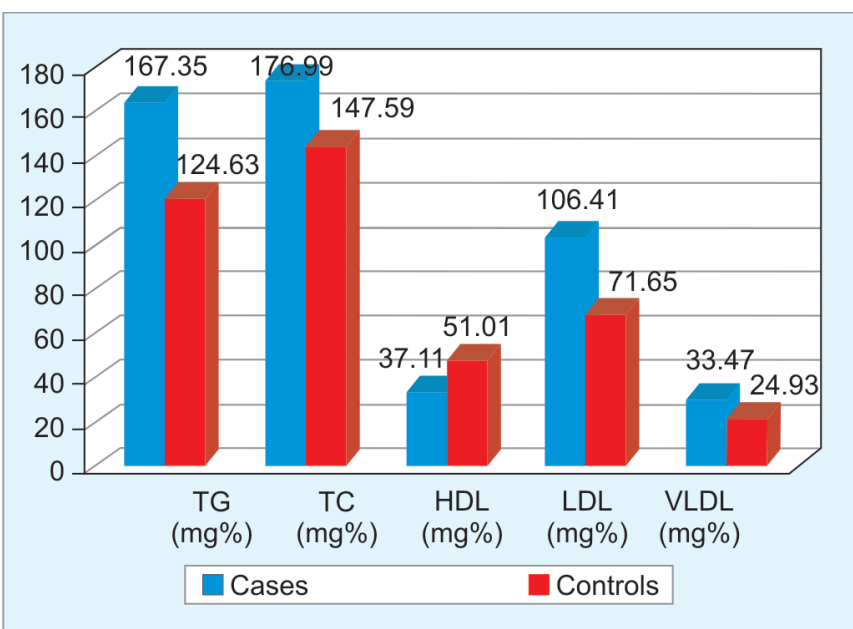

Fig. 2: Graphical comparison of lipid profile parameters

Table 3: Correlation between hs-CRP and lipid profile

\begin{tabular}{llllll}
\hline & $T G$ & $T C$ & $H D L$ & $L D L$ & $V L D L$ \\
\hline hs-CRP $r$ value & 0.29 & 0.41 & -0.29 & 0.4 & 0.29 \\
hs-CRP $p$ value & $<0.05$ & $<0.001^{*}$ & $<0.05$ & $<0.001^{*}$ & $<0.05$ \\
\hline
\end{tabular}

*Statistically significant i.e., $p<0.001$

and interleukin-6 (IL-6), have been linked to IR, and their expression is increased in adipose tissue.

Total 100 nondiabetic subjects with family history of T2DM were investigated for plasma glucose, serum hs-CRP, and lipid profile, and compared with 100 healthy controls.

This study was undertaken to compare in the cases and controls and the relationship of hs-CRP with lipid profile. We also aimed at correlating various biochemical and demographic parameters with each other.

\section{Demographic Variables}

- Mean values of age, weight, and height were not different in the cases and controls ( $p>0.05$ ).

- Waist/hip ratio was higher in the cases $(0.81 \pm 0.07$ vs $0.77 \pm$ $0.04, p<0.001$ ) than controls.

- Waist/hip ratio also showed positive correlation with BMI ( $r=$ $0.43, p<0.05)$.

The presence of visceral obesity could lead to increased production of cytokines, such as IL- 6 and TNF- $a$, by visceral adipocytes, which could induce higher hs-CRP production by liver. ${ }^{10}$ Storage of energy as fat, especially as intra-abdominal fat, is a more efficient way of storing energy than as glycogen in muscle and liver. This is what we find in the insulin-resistant phenotype and explains why offspring of subjects with T2DM show early accumulation of abdominal fat. ${ }^{5}$

Our study was in agreement with study by Papazafiropoulou et al. who found that subjects with a diabetic parent had higher BMI $\left(31.22 \pm 5.87\right.$ vs $\left.30.67 \pm 5.35 \mathrm{~kg} / \mathrm{m}^{2}, p=0.08\right)$ and higher prevalence of dyslipidemia ( 49.8 vs $44.6 \%, p=0.06$ ) when compared with subjects with no diabetic relatives. ${ }^{11}$

High-sensitivity C-reactive protein levels are positively influenced more by $\mathrm{W} / \mathrm{H}$ ratio than BMI. Visceral adipocytes plays key role in regulating inflammation. C-reactive protein is synthesized in the liver and is regulated by proinflammatory cytokines, such as IL- 6 and TNF- $a$. This suggests that, association of increased hs-CRP concentration with increased insulin levels could be due to the presence of chronic systemic subclinical inflammation. ${ }^{6}$

C-reactive protein bound to membranes of damaged vascular cells activates complement proteins. ${ }^{12}$ Inflammation disturbs pancreatic $\beta$-cell function in genetically susceptible individuals who are at high risk to develop diabetes. Inflammation could also promote hepatic IR in individuals who are genetically susceptible to develop diabetes. Insulin resistance exacerbates fasting glycemia and hypertriglyceridemia. ${ }^{13}$

In the FDRs of T2DM subjects, increased IL- 6 and TNF- $a$ reduces the expression of IRS-1, GLUT-4, and decreases insulin-stimulated glucose transport. ${ }^{14}$

Hojbjerre et al. found IR, elevated plasma C-reactive protein, leptin, and monocyte chemoattractant protein-1 (MCP-1), high IL-6 in the FDRs of T2DM subjects. ${ }^{14}$ Zehua et al. found increased hs-CRP $(p<0.05)$, fasting plasma glucose $(p<0.01)$ in normoglycemic FDRs of T2DM patients. ${ }^{15}$ Kriketos et al. found $20 \%$ lower glucose infusion rate $(\mathrm{GIR})(51.8 \pm 3.9 \mathrm{vs} 64.9 \pm 4.6 \mu \mathrm{mol} /$ minute $/ \mathrm{kg}$ fat-free mass, $p=$ 0.04 ) which was measure of insulin sensitivity in the FDRs of T2DM than controls. However, FDR of T2DM subjects had normal and comparable levels of CRP, adiponectin, and complement proteins with controls without family history of diabetes. C-reactive protein was inversely related to $\operatorname{GIR}(r=0.33, p=0.04)$ and adiponectin $(r=$ $0.34, p=0.03)$ and positively related to adiposity $(p=0.04)$. However, CRP was not related to GIR independent of fat mass. ${ }^{16}$ Our results suggest that T2DM associated with a state of chronic low-grade systemic inflammation indicated by raised hs-CRP levels could occur early in the disease course, even if the FDRs are normoglycemic.

Gulcelik et al. found significantly higher CRP levels in the FDRs of T2DM patients with IGT test $(4.12 \pm 1.7 \mathrm{mg} / \mathrm{L})$ than in the FDR of T2DM patients with NGT test $(3.7 \pm 2.1 \mathrm{mg} / \mathrm{L})$ and controls $(2.1 \pm$ $0.8 \mathrm{mg} / \mathrm{L})$, all $p<0.05 .^{17}$

Possible explanations for significant relationship between hs-CRP and lipid profile parameters are as follows:

- Dyslipidemia may be genetically determined and that genetic determinants could be related to family history of T2DM. ${ }^{18}$

- It is possible that some FDRs of T2DM patients have larger intraabdominal fat store that might contribute to the development of lipid abnormalities. ${ }^{19}$

Impaired postprandial suppression of hepatic release of VLDL by insulin may be the reason for lipid abnormalities in the FDRs of T2DM subjects. Phosphoinositol-3-kinase which is key enzyme for initiating insulin action is necessary for suppression for the release of VLDL. Impaired cellular activation of PI-3 kinase by insulin may be another reason for dyslipidemia in offspring of T2DM subjects. ${ }^{20}$ 
Our study correlated with a study by Axelsen et al. in which they found IR in spite of normal glucose tolerance in the FDRs of T2DM subjects. Relatives also showed postprandial hypertriglyceridemia $(p=0.037) .{ }^{21}$ Tian et al. found high TG, low HDL levels in the FDRs of T2DM subjects than subjects without family history of T2DM. ${ }^{22}$ Perseghin et al. found higher free fatty acid (FFA) levels (582 \pm 189 vs $470 \pm 140 \mu \mathrm{mol} ; p=0.007$ ) whereas TG, TC, HDL, and LDL were comparable with those of control subjects. ${ }^{23}$ Edavan et al. found low HDL $(p<0.001)$ among normoglycemic offspring of individuals with T2DM. TG $(p=0.251)$, TC $(p=0.322)$, and VLDL $(p=0.46)$ were comparable to controls with no family history of diabetes. ${ }^{24}$

In the present study, hs-CRP was found to be positively correlated with TG $(r=0.29, p<0.05)$, TC $(r=0.41, p<0.001)$, LDL $(r=0.4, p<0.05)$, and VLDL $(r=0.29, p<0.05)$ suggesting role of inflammation in deranged lipid profile and hs-CRP was found to be negatively correlated with $\mathrm{HDL}(r=-0.29, p<0.05)$. Our study correlated well with the study by Deng et al. in which they found levels of hs-CRP were high $(p=0.01)$ in the FDRs than control. Levels of hs-CRP were correlated with low LDL $(r=0.3, p<0.05)$ and TC ( $r$ $=0.26, p<0.05) .{ }^{25}$ Vikram et al. did not found significant correlation of hs-CRP with fasting insulin and lipid parameters. ${ }^{26}$

The possible expiations for role of hs-CRP in deranged lipid profile are as follows:

- The primary cytokine involved in the hepatic CRP synthesis is IL-6.

- IL- 6 inhibits lipoprotein lipase activity and increases concentration of non-esterified fatty acids by which IL- 6 modifies adipocyte glucose and lipid metabolism. ${ }^{27}$

From the above findings, we can conclude that in the FDRs of T2DM patients, a state of chronic low-grade systemic inflammation may be present leading to early abnormalities in lipid profile parameters before the glucose tolerance becomes abnormal.

In the present study, we noticed significant hs-CRP shows positive correlation with TG, TC, LDL, and VLDL and has negative correlation with HDL.

\section{Conclusion}

High-sensitivity C-reactive protein levels were increased in the FDRs of T2DM subjects suggesting that IR is a state of chronic lowgrade systemic inflammation. All lipid profile parameters were significantly deranged in the FDRs of T2DM subjects documenting that dyslipidemia may occur early in the course of disease in the FDR of T2DM.

Timely screening and early detection of the increased hs-CRP in the FDRs of T2DM subjects may help clinicians enable to intervene early in the course of disease and prevent further complications and outcomes. Therefore, primary prevention by target screening among high-risk individuals to prevent transition to overt T2DM by therapeutic lifestyle changes is a feasible and attractive alternative to reduce diabetes-related morbidity and mortality.

\section{References}

1. Ramachandran A, Snehalatha C. Current scenario of diabetes in India. J Diabetes 2009;1(1):18-28. DOI: 10.1111/j.1753-0407.2008.00004.x.

2. Mohan V, Sandeep S, Deepa R, et al. Epidemiology of type II diabetes: Indian scenario. Indian J Med Res 2007;125(3):217-230.

3. Alberti $G$, Zimmet $P$, Shaw $P$, et al. Type II diabetes in the young: the evolving epidemic. Diabetes Care 2004;27(7):1798-1811. DOI: 10.2337/ diacare.27.7.1798.
4. World Health Organization. Report of a WHO consultation, definition, diagnosis and classification of diabetes mellitus and its complication: WHO, department of non-communicable disease surveillance. Geneva 1999. 1-59.

5. Ridderstrale M, Groop L. Genetic dissection of type II diabetes. Mol Cell Endocrinol 2009;297(1-2):10-17. DOI: 10.1016/j.mce.2008.10.002.

6. Ramachandran A, Snehalatha C. Type II diabetes mellitus-the epidemic of the 21st century: the Indian scenario. Int J Diab Dev Countries 1999;19:158-164.

7. Carmena R. High risk of lipoprotein dysfunction in type II diabetes mellitus. Rev Esp Cardiol 2008;8(Supl C):18-24.

8. Goldberg IJ. Diabetic dyslipidemia: causes and consequences. J Clin Endocrinol Metab 2001;86(3):965-971. DOI: 10.1210/jcem.86.3.7304.

9. Shishehmbor $\mathrm{MH}$, Bhatt DL, Topol EJ, et al. Using C-reactive protein to asses cardiovascular disease risk. ClevClin J Med 2003;70(7):634-640.

10. Chandalia M, Cabo-Chan AV, Devaraj S, et al. Elevated plasma highsensitivity C-reactive protein concentrations in asian indians living in the United States. J Clin Endocrinol Metab 2003;88(8):3773-3776. DOI: 10.1210/jc.2003-030301.

11. Papazafiropoulou A, Sotiropoulos A, Skliros E, et al. Familial history of diabetes and clinical characteristics in greek subjects with type II diabetes. BMC Endocr Disord 2009;9:12. DOI: 10.1186/1472-68239-12.

12. Gelaye B, Revilla L, Lopez T, et al. Association between insulin resistance and $\mathrm{C}$-reactive protein among peruvian adults. Diabetol Metab Syndr 2010;2(1):30. DOI: 10.1186/1758-5996-2-30.

13. Laaksonen DE, Niskanen L, Nyyssonen K, et al. C-reactive protein and the development of the metabolic syndrome and diabetes in middle-aged men. Diabetologia 2004;47(8):1403-1410. DOI: 10.1007/ s00125-004-1472-x.

14. Hojbjerre L, Sonne MP, Alibegovic AC, et al. Impact of physical inactivity on adipose tissue low-grade inflammation in first-degree relatives of type II diabetics. Diabetes Care 2011;34(10):2265-2272. DOI: 10.2337/dc11-0631.

15. Zehua Z, Wang ZH, Zhang SU, et al. High sensitive C-reactive protein level and its clinical significance in normoglycemic first-degree relatives of type II diabetic family. J Pract Med 2009.

16. Kriketos AD, Greenfield JR, Peake PW, et al. Inflammation, insulin resistance, and adiposity: a study of first-degree relatives of type II diabetic subjects. Diabetes Care 2004;27(8):2033-2040. DOI: 10.2337/ diacare.27.8.2033.

17. Gulcelik NE, Serter R, Ozkaya M, et al. Association of C-reactive protein with insulin resistance in first degree relatives of diabetic patients. Endocrine 2006;11:329.

18. Eriksson JW, Buren J, Svensson M, et al. Postprandial regulation of blood lipids and adipose tissue lipoprotein lipase in type II diabetes patients and healthy control subjects. Atherosclerosis 2003;166(2):359-367. DOI: 10.1016/S0021-9150(02)00366-0.

19. Sandeep S, Gokulakrishnan K, Velmurugan K, et al. Visceral and subcutaneous abdominal fat in relation to insulin resistance and metabolic syndrome in non-diabetic south Indians. Indian J Med Res 2010;131:629-635.

20. Taskinen MR. Diabeticdyslipidemia: from basic research to clinical practice. Diabetologia 2003;46(6):733-749. DOI: 10.1007/s00125-0031111-y.

21. Axelsen M, Eriksson JW, Taskinen MR, et al. Postprandial hypertriglyceridemia and insulin resistance in normoglycemic first-degree relatives of patients with type II diabetes. Ann Intern Med 1999;131(1):27-31. DOI: 10.7326/0003-4819-131-1-19990706000006.

22. Tian H, Han L, Ren Y, et al. Lipoprotein (a) level and lipids in type II diabetic patients and their normoglycemic first-degree relatives in type II diabetic pedigrees. Diabetes Res Clin Pract 2003;59(1):63-69. DOI: 10.1016/S0168-8227(02)00158-4.

23. Perseghin G, Ghosh S, Gerow K, et al. Metabolic defects in lean nondiabetic offspring of NIDDM parents: a cross-sectional study. Diabetes 1997;46(6):1001-1009. DOI: 10.2337/diab.46.6.1001. 
24. Edavan P, Kulshreshtha B, Khurana ML, et al. Low HDL-cholesterol among normal weight, normoglycemic offspring of individuals with type II diabetes mellitus. Hormones 2011;10(1):57-66. DOI: 10.14310/ horm.2002.1293.

25. Deng CX, Ling G, Rong ZH. Relation of high-sensitivity C-reactive protein with insulin resistance in the first-degree relatives of type II diabetics. Chin J Diabetes 2008.
26. Vikram NK, Misra A, Pandey RM, et al. Association between subclinical inflammation and fasting insulin in urban young adult north Indian males. IJMR 2006;124(6):677-682.

27. Pradhan AD, Manson JE, Rifai N, et al. C-reactive protein, interleukin 6 , and risk of developing type II diabetes mellitus. JAMA 2001;286(3):327-334. DOI: 10.1001/jama.286. 3.327. 